\title{
PARAMETRIC SENSITIVITY ANALYSIS: A CASE STUDY IN OPTIMAL CONTROL OF FLIGHT DYNAMICS
}

\author{
Christof Büskens \\ Lehrstuhl für Ingenieurmathematik, Universität Bayreuth \\ Universitätsstr. 30, D-95440 Bayreuth, Germany \\ christof.bueskens@uni-bayreuth.de
}

\author{
Kurt Chudej \\ Lehrstuhl für Ingenieurmathematik, Universität Bayreuth \\ Universitätsstr. 30, D-95440 Bayreuth, Germany \\ kurt.chudej@uni-bayreuth.de
}

\begin{abstract}
Realistic optimal control problems from flight mechanics are currently solved by sophisticated direct or indirect methods in a fast and reliable way. Often one is not only interested in the optimal solution of one control problem, but is also strongly interested in the sensitivity of the optimal solution due to perturbations in certain parameters (constants or model functions) of the process. In the past this problem was solved by time-consuming parameter studies: A large number of almost similar optimal control problems were solved numerically. Sensitivity derivatives were approximated by finite differences. Recently a new approach, called parametric sensitivity analysis, was adapted to the direct solution of optimal control processes [3]. It uses the information gathered in the optimal solution of the unperturbed (nominal) optimal control problem to compute sensitivity differentials of all problem functions with respect to these parameters. This new approach is described in detail for an example from trajectory optimization.
\end{abstract}

Keywords: parametric sensitivity analysis, optimal control, direct methods, trajectory optimization.

\section{Introduction}

Realistically modelled optimal control problems can be solved efficiently and reliably by sophisticated direct and indirect methods (see

The original version of this chapter was revised: The copyright line was incorrect. This has been corrected. The Erratum to this chapter is available at DOI: 10.1007/978-0-387-35699-0_19 
e.g. the survey articles [1], [10]). Trajectory optimization problems for aircrafts and space vehicles usually pose hard challenges for the direct and indirect solution algorithms. A couple of direct algorithms have proved their ability to solve accurately and reliably trajectory optimization problems in the last decade, such as e.g. SOCS (Betts [2]), GESOP (Jänsch, Well, Schnepper [9]), DIRCOL (von Stryk [12]) and NUDOCCCS (Büskens [3]). Trajectory optimization problems use in general complicated models of the surrounding atmospheric effects, the performance and consumption of the engines and the ability to maneuver. Usually optimal solutions are computed at first for a nominal data set of the model. Later huge parameter studies are done for perturbed model data. This means that the whole optimization process is started again for the huge number of perturbed models.

We present a new approach of Büskens [3]: Exploiting already computed information during the solution of the nominal optimal control problem to derive sensitivity information. This substitutes the additional solution of perturbed optimal control problems.

We explain the new approach of parametric sensitivity analysis in detail for an example from flight mechanics. The trajectory optimization problem is concerned with minimizing the amount of fuel used per travelled range over ground with periodic boundary conditions. It is interesting that by periodic trajectories and controls savings in fuel consumption can be achieved in comparison to the steady-state solution.

In order to normalize the changing effects of the atmosphere due to the weather, one uses data of a reference atmosphere in the computational model. Unfortunately there exist a couple of reference atmospheres. Additionally one is also interested in realistic changes of the air density onto the computed optimal solution.

We therefore provide not only the nominal solution but also the sensitivity with respect to the air density as an example. Note that no parameter studies are needed. Information gathered during the computation of the nominal solution is used.

Applications to further perturbation parameters in the model are straight forward.

\section{A Trajectory Optimiziation Problem}

Aircraft usually use steady-state cruise to cover long distances. It is interesting, that these steady-state trajectories are non-optimal with respect to minimizing fuel [11].

The following optimal control problem from [8], enlarged by a perturbation parameter $p$, describes the problem of minimizing fuel per 
travelled range over ground for a realistically modelled aircraft flying in a vertical plane.

State variables are velocity $v$, flight path angle $\gamma$, altitude $h$ and weight $W$. The range $x$ is used as the independent variable. The lift coefficient $C_{L}$ and the throttle setting $\delta$ are the control variables.

For a given value of the perturbation parameter $p$ (nominal value is here $\left.p_{0}=1\right)$ find control functions $C_{L}(x ; p)$ and $\delta(x ; p)$ and the final range $x_{\mathrm{f}}(p)$ such that the cost functional

$$
I=\left[W_{0}-W\left(x_{\mathrm{f}}\right)\right] / x_{\mathrm{f}}
$$

is minimized and the following equations of motion, control constraints and boundary conditions are fulfilled.

$$
\begin{aligned}
& \frac{d v}{d x}=\frac{g}{v \cos \gamma}\left[\frac{T(h, M) \delta-D\left(h, M, C_{L}\right)}{W_{0}}-\sin \gamma\right] \\
& \frac{d \gamma}{d x}=\frac{g}{v^{2}}\left[\frac{L\left(h, M, C_{L}\right)}{W_{0} \cos \gamma}-1\right] \\
& \frac{d h}{d x}=\tan \gamma \\
& \frac{d W}{d x}=-T(h, M) \delta \frac{c(h, M)}{v \cos \gamma} \\
& \begin{array}{c}
0 \leq C_{L} \leq C_{L, \max } \\
\delta_{\min } \leq \delta \leq 1
\end{array} \\
& v(0)=v\left(x_{\mathrm{f}}\right), \gamma(0)=\gamma\left(x_{\mathrm{f}}\right), h(0)=h\left(x_{\mathrm{f}}\right), W(0)=W_{0}
\end{aligned}
$$

Model functions are the Mach number $M$, speed of sound $a$, thrust $T$, consumption $c$, lift $L, \operatorname{drag} D$, air density $\rho . S$ denotes the constant reference area. $g$ denotes the gravitational constant.

$$
\begin{array}{ll}
M(v, h) & =v / a(h) \\
a(h) & =\alpha_{4} \sqrt{\sum_{i=0}^{3} \alpha_{i} h^{i}} \\
T(h, M) & =c_{1}(h)+c_{2}(h) M+c_{3}(h) M^{2}+c_{4}(h) M^{3} \\
c(h, M) & =d_{1}(h)+d_{2}(h) M+d_{3}(h) M^{2}+d_{4}(h) M^{3} \\
L\left(v, h, C_{L}\right) & =\rho(h) S v^{2} C_{L} / 2 \\
D\left(v, h, C_{L}\right) & =\rho(h) S v^{2}\left[C_{D 0}(M)+\Delta C_{D}\left(M, C_{L}\right)\right] / 2 \\
C_{D 0}(M) & =a_{1} \arctan \left[a_{2}\left(M-a_{3}\right)\right]+a_{4} \\
\Delta C_{D}\left(M, C_{L}\right) & =b_{1}(M) C_{L}^{2}+b_{2}(M) C_{L}^{4}+b_{3}(M) C_{L}^{6}+b_{4}(M) C_{L}^{8} \\
\rho(h) & =p \rho_{0} \exp \left[\beta_{7}+\beta_{6} h+\beta_{5} \exp \left(\sum_{i=1}^{4} \beta_{i} h^{i}\right)\right]
\end{array}
$$


The coefficients of the polynomials $b_{i}(M), c_{i}(h), d_{i}(h)$, and the constants $\rho_{0}, \beta_{i}, a_{i}, S, \alpha_{i}, W_{0}, \delta_{\min }, C_{L, \max }$ can be found in the [8].

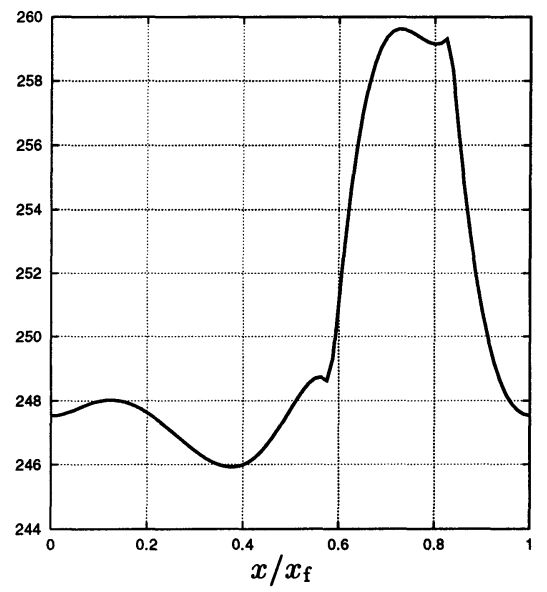

Figure 1. Nominal optimal state $v\left(x ; p_{0}\right)$

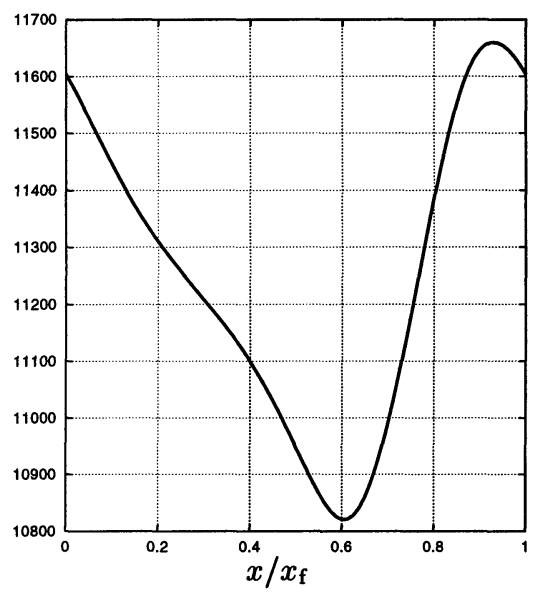

Figure 3. Nominal optimal state $h\left(x ; p_{0}\right)$

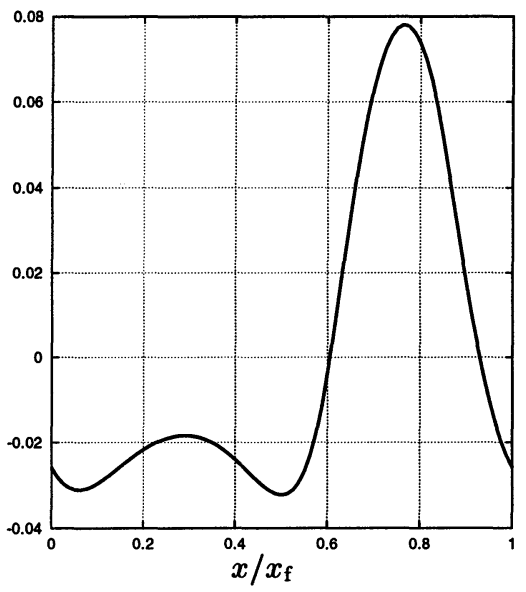

Figure 2. Nominal optimal state $\gamma\left(x ; p_{0}\right)$

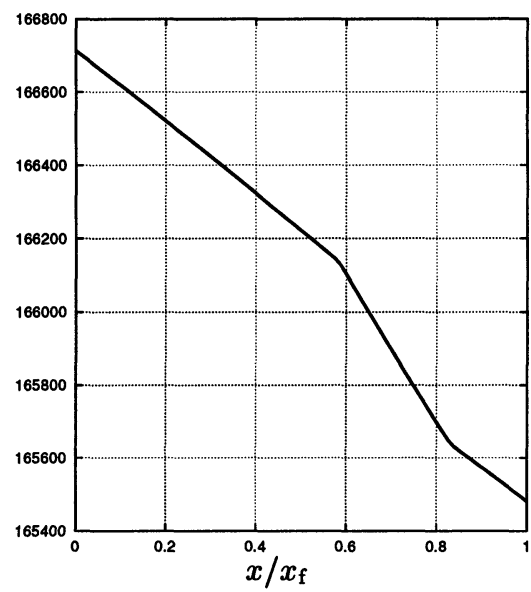

Figure 4. Nominal optimal state $W\left(x ; p_{0}\right)$

As an additional perturbation parameter we use $p$. A solution by an indirect multiple shooting algorithm is presented in [8] for the nominal value of $p_{0}=1$. In these times, before sophisticated direct methods were developed, elaborated homotopies were required for the indirect 


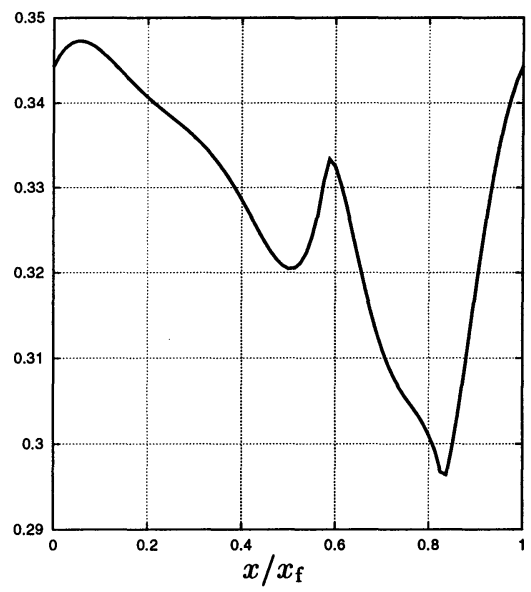

Figure 5. Nominal optimal control $C_{L}\left(x ; p_{0}\right)$

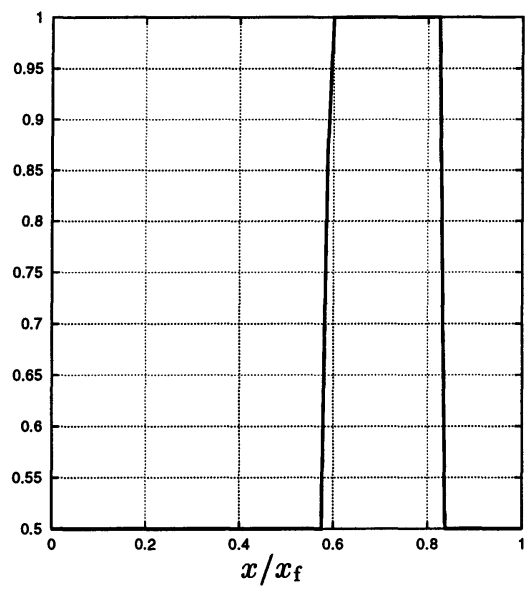

Figure 6. Nominal optimal control $\delta\left(x ; p_{0}\right)$

solution. Today these initial estimates for the indirect method can be provided easily by direct methods. The figures 1-4 show the optimal states and the figures 5-6 show the optimal controls for the nominal value of $p=p_{0}=1$ computed by the direct method NUDOCCCS (Büskens [3]).

Moreover the direct method NUDOCCCS can compute in a postprocessing step also the sensitivities of the optimal states $\frac{\partial v}{\partial p}(x ; p), \ldots$, $\frac{\partial W}{\partial p}(x ; p)$ and the optimal controls $\frac{\partial C_{L}}{\partial p}(x ; p), \frac{\partial \delta}{\partial p}(x ; p)$ with respect to perturbation parameters $p$.

\section{Parametric Sensitivity Analysis}

The general mathematical approach for a parametric sensitivity analysis of perturbed optimal control problems is based on NLP methods:

The following autonomous perturbed control problem of Mayer-form will be referred to as problem $\mathbf{O C P}(\mathbf{p})$ :

For a given perturbation parameter $p \in P$ find control functions $u(x ; p)$ and the final time $x_{\mathrm{f}}(p)$ such that the cost functional

$$
J=g\left(y\left(x_{\mathrm{f}}\right), p\right)
$$

is minimized subject to the following constraints

$$
\begin{array}{lll}
y^{\prime}(x)=f(y(x), u(x), p), & x \in\left[0, x_{\mathrm{f}}\right] \\
\psi\left(y(0), y\left(x_{\mathrm{f}}\right), p\right)=0 & , & \\
C(y(x), u(x), p) \leq 0 & , & x \in\left[0, x_{\mathrm{f}}\right] .
\end{array}
$$


Herein $y(x) \in \mathbb{R}^{n}$ denotes the state of a system and $u(x) \in \mathbb{R}^{m}$ the control with respect to an independent variable $x$, which is often the time.

In the previously introduced trajectory optimization problem the independent variable $x$ denotes the range, the state is given by $y:=(v, \gamma, h, W)^{\top}$ and the control by $u:=\left(C_{L}, \delta\right)^{\top}$.

The functions $g: \mathbb{R}^{n} \times P \rightarrow \mathbb{R}, f: \mathbb{R}^{n+m} \times P \rightarrow \mathbb{R}^{n}, \psi: \mathbb{R}^{2 n} \times P \rightarrow$ $\mathbb{R}^{r}$, and $C: \mathbb{R}^{n+m} \times P \rightarrow \mathbb{R}^{k}$ are assumed to be sufficiently smooth on appropriate open sets. The final time $x_{\mathrm{f}}$ is either fixed or free. Note that the formulation of mixed control-state constraints $C(y(x), u(x), p) \leq 0$ in (5) includes pure control constraints $C(u(x), p) \leq 0$ as well as pure state constraints $C(y(x), p) \leq 0$. It is well known, that problems of form $\mathbf{O C P}(\mathbf{p})$ can be solved efficiently by approximating the control functions $u^{i} \approx u\left(x_{i}\right)$ for given mesh points $x_{i} \in\left[0, x_{\mathrm{f}}\right], i=1, \ldots, N$ and solving the state variables by standard integration methods. This leads to approximations $y\left(x_{i} ; z, p\right) \approx y\left(x_{i}\right), z:=\left(u^{1}, \ldots, u^{N}\right)$ of the state at the mesh points $x_{i}$. For a more detailed discussion please refer to [3][6].

Therefore the optimal control problem $\mathbf{O C P}(\mathbf{p})$ is replaced by the finite dimensional perturbed nonlinear optimization problem $\operatorname{NLP}(\mathbf{p})$

$$
\begin{gathered}
\text { For a given } p \in P \\
\min _{z} g\left(y\left(x_{N} ; z, p\right), p\right) \quad \text { s.t. } \\
\psi\left(y\left(x_{N} ; z, p\right), p\right)=0, \\
C\left(y\left(x_{i} ; z, p\right), u^{i}, p\right) \leq 0, \quad i=1, \ldots, N .
\end{gathered}
$$

Several reliable optimization codes have been developed for solving NLP problems (6), like e.g. SQP methods. This idea is implemented e.g. in the direct methods SOCS, GESOP, DIRCOL and NUDOCCCS.

An additional, and to our knowledge unique, feature of NUDOCCCS (Büskens [3]) is the ability to compute accurately the sensitivity differentials $\frac{\partial y}{\partial p}\left(x ; p_{0}\right), \frac{\partial u}{\partial p}\left(x ; p_{0}\right)$ of the approximations

$$
\begin{aligned}
& y\left(x ; p_{0}+\Delta p\right) \approx y\left(x ; p_{0}\right)+\frac{\partial y}{\partial p}\left(x ; p_{0}\right) \cdot \Delta p \\
& u\left(x ; p_{0}+\Delta p\right) \approx u\left(x ; p_{0}\right)+\frac{\partial u}{\partial p}\left(x ; p_{0}\right) \cdot \Delta p .
\end{aligned}
$$

This is done by the following idea:

Let $z_{0}$ denote the unperturbed solution of $\operatorname{NLP}\left(\mathbf{p}_{\mathbf{0}}\right)$ for a nominal parameter $p=p_{0}$ and let $h^{a}$ denote the collection of active constraints in (6).

$$
L(z, \mu, p):=g\left(y\left(x_{N} ; z, p\right), p\right)+\mu^{\top} h^{a}(z, p)
$$

is the Lagrangian function with the associated Lagrange multiplier $\mu$. Then the following results hold [7]: 
Solution Differentiability for NLP-problems: Suppose that the optimal solution $\left(z_{0}, \mu_{0}\right)$ for the nominal problem $\mathbf{N L P}\left(\mathbf{p}_{\mathbf{0}}\right)$ satisfies a maximal rank condition for $h_{z}^{a}\left(z_{0}, p_{0}\right)$, second order sufficient optimality conditions and strict complementarity of the multiplier $\mu$. Then the unperturbed solution $\left(z_{0}, \mu_{0}\right)$ can be embedded into a $C^{1}$-family of perturbed solutions $(z(p), \mu(p))$ for $\mathbf{N L P}(\mathbf{p})$ with $z\left(p_{0}\right)=z_{0}, \mu\left(p_{0}\right)=\mu_{0}$.

The sensitivity differentials of the optimal solutions are given by the formula

$$
\left(\begin{array}{c}
\frac{d z}{d p}\left(p_{0}\right) \\
\frac{d \mu}{d p}\left(p_{0}\right)
\end{array}\right)=-\left(\begin{array}{cc}
L_{z z} & \left(h_{z}^{a}\right)^{\top} \\
h_{z}^{a} & 0
\end{array}\right)^{-1}\left(\begin{array}{c}
L_{z p} \\
h_{p}^{a}
\end{array}\right)
$$

evaluated at the optimal solution. This formula provides good approximations for the sensitivity of the perturbed optimal controls at the mesh points, i.e. for the quantities $\frac{\partial u}{\partial p}\left(x_{i} ; p_{0}\right), i=1, \ldots, N$. Then the state sensitivities $\frac{\partial y}{\partial p}\left(x_{i} ; p_{0}\right)$ are obtained by differentiating the control-state relation in (6) $y\left(x_{i}\right)=y\left(x_{i}, z, p\right)$ with respect to the parameter $p$ :

$$
\frac{\partial y}{\partial p}\left(x_{i} ; p_{0}\right) \approx \frac{\partial y}{\partial z}\left(x_{i} ; z_{0}, p_{0}\right) \frac{d z}{d p}\left(p_{0}\right)+\frac{\partial y}{\partial p}\left(x_{i} ; z_{0}, p_{0}\right) .
$$

The sensitivity differentials of the adjoint variables or objective functional can be calculated respectively.

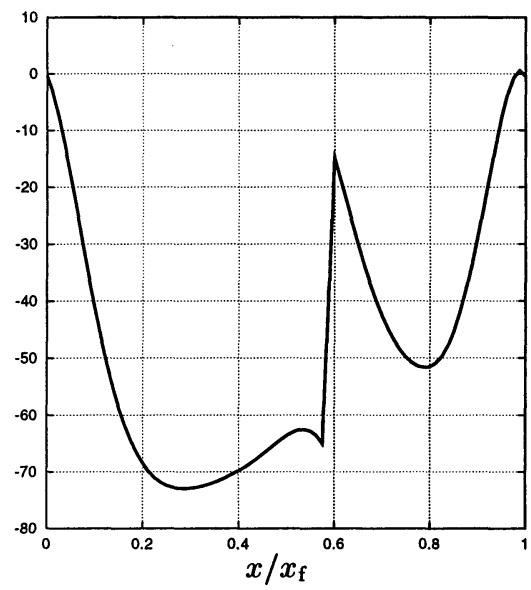

Figure 7. Sensitivity $\partial v / \partial p\left(x ; p_{0}\right)$

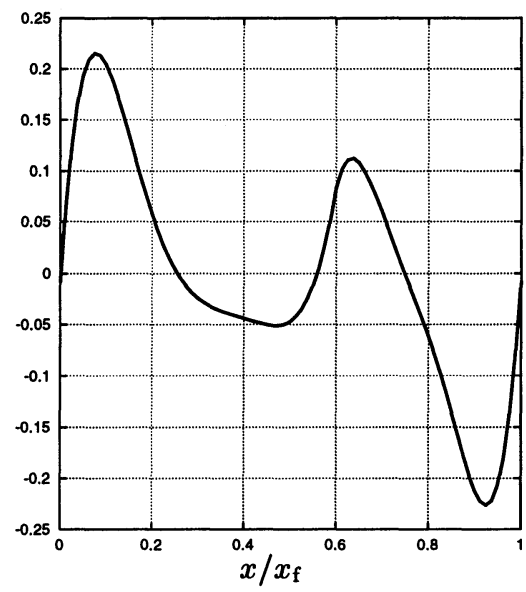

Figure 8. Sensitivity $\partial \gamma / \partial p\left(x ; p_{0}\right)$

We return back to the example. In the first step the optimal nominal solution is calculated by the code NUDOCCCS of Büskens [3], see figures 1-6. In the second step the sensitivity differentials of the model functions (states, controls, adjoint variables, cost functional and further 
interesting model functions) are calculated from equations $(8,9)$, see figures $7-12$.

These figures provide valuable information for the engineers. Additional perturbation parameters can be added to the model. Basically only an additional matrix vector multiplication is needed in order to compute the sensitivity differentials of the states and controls for each component of the perturbation parameter.

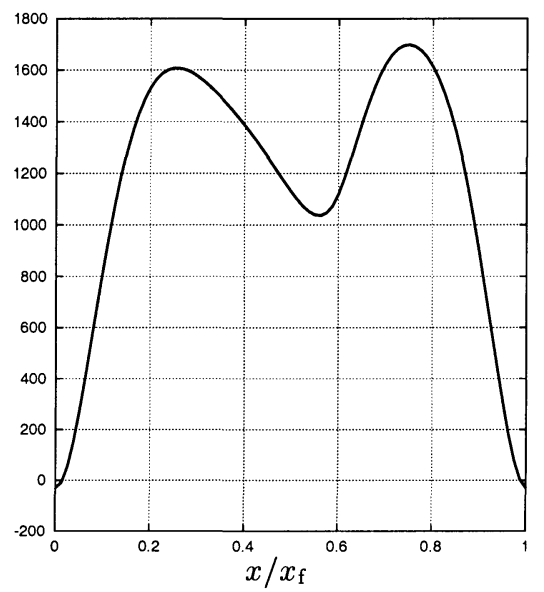

Figure 9. Sensitivity $\partial h / \partial p\left(x ; p_{0}\right)$

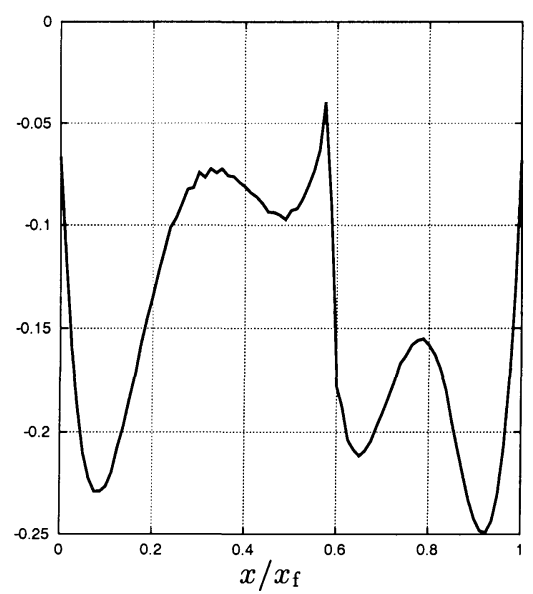

Figure $11 . \quad$ Sensitivity $\partial C_{L} / \partial p\left(x ; p_{0}\right)$

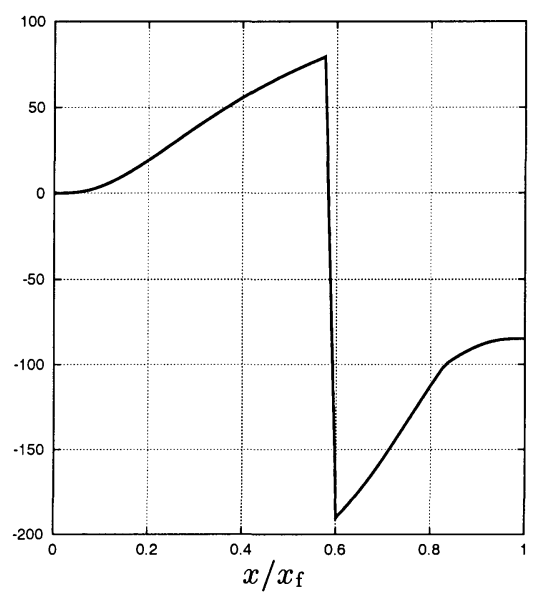

Figure 10. Sensitivity $\partial W / \partial p\left(x ; p_{0}\right)$

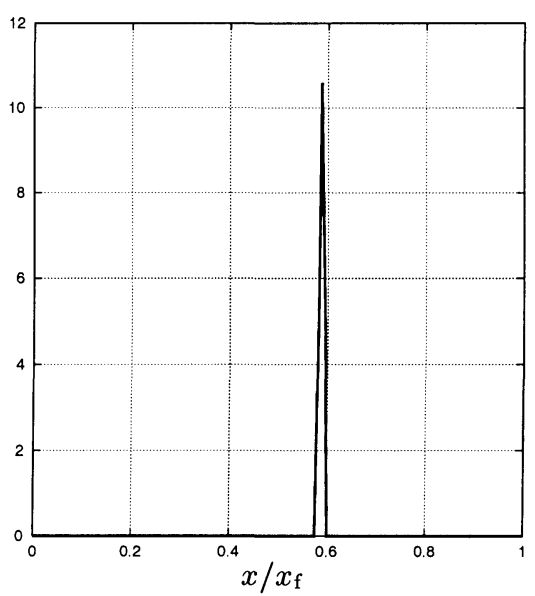

Figure 12. Sensitivity $\partial \delta / \partial p\left(x ; p_{0}\right)$ 


\section{References}

[1] Betts, J. T. (1998) Survey of Numerical Methods for Trajectory Optimization. Journal of Guidance, Control, and Dynamics, Vol. 21, pp. 193-207.

[2] Betts, J. T. (2001) Practical Methods for Optimal Control Using Nonlinear Programming. SIAM, Philadelphia.

[3] Büskens, C. (1998) Optimierungsmethoden und Sensitivitätsanalyse für optimale Steuerprozesse mit Steuer- und Zustands-Beschränkungen. Dissertation, Universität Münster.

[4] Büskens, C., Maurer, H. (2000) SQP-Methods for Solving Optimal Control Problems with Control and State Constraints: Adjoint Variables, Sensitivity Analysis and Real-Time Control. Journal of Computational and Applied Mathematics, Vol. 120, pp. 85-108.

[5] Büskens, C., Maurer, H. (2001) Sensitivity Analysis and Real-Time Optimization of Parametric Nonlinear Programming Problems. - In: Grötschel, M., Krumke, S.O., Rambau, J. (Eds.): Online Optimization of Large Scale Systems: State of the Art. Springer Verlag, Berlin, pp. 3-16.

[6] Büskens, C., Maurer, H. (2001) Sensitivity Analysis and Real-Time Control of Parametric Optimal Control Problems Using Nonlinear Programming Methods. - In: Grötschel, M., Krumke, S.O., Rambau, J. (Eds.): Online Optimization of Large Scale Systems: State of the Art. Springer Verlag, Berlin, pp. 57-68.

[7] Fiacco, A.V. (1983) Introduction to Sensitivity and Stability Analysis in Nonlinear Programming. Academic Press, New York.

[8] Grimm, W., Well, K.H., Oberle, H.J. (1986) Periodic Control for Minimum-Fuel Aircraft Trajectories. Journal of Guidance, Vol. 9, 169-174.

[9] Jänsch, C., Well, K.H., Schnepper, K. (1994) GESOP - Eine Software Umgebung zur Simulation und Optimierung. In: Proc. des SFB 255 Workshops Optimalsteuerungsprobleme von Hyperschall-Flugsystemen, Ernst-Moritz-Arndt Universität Greifswald, pp. 15-23.

[10] Pesch, H.J. (1994) A Practical Guide to the Solution of Real-Life Optimal Control Problems. Control and Cybernetics, 23, pp. 7-60.

[11] Speyer, J.L. (1976) Nonoptimality of the Steady-State Cruise for Aircraft. AIAA Journal, Vol. 14, pp. 1604-1610.

[12] von Stryk, O. (1995) Numerische Lösung optimaler Steuerungsproblems: Diskretisierung, Parameteroptimierung und Berechnung der adjungierten Variablen. VDI-Verlag, Reihe 8, Nr. 441. 